\section{DIAGNOSIS IN THE MANAGEMENT OF BURNS*}

BY

\author{
D. MacG. JACKSON, M.D., F.R.C.S. \\ Burns Unit, Birmingham Accident Hospital
}

An extensive burn is an incident which starts a fastmoving progressive illness. The natural history of the disease is known. Within an hour or two a severe burn will produce oligaemia, clinical shock, and sometimes renal failure. Bacterial colonization of the burn surface follows, and sometimes invasive infection. Soon anaemia and wasting become apparent, and in a few weeks the formation of fibrous tissue may cause contractures and deformity. Against this background the need for constant re-diagnosis of the patient's changing condition is imperative to prevent or arrest the expected complications.

To achieve more accurate assessment a constant endeavour is being made to introduce into the early stages of emergency treatment new diagnostic methods of proved value. Although special investigations are difficult to fit into the tempo of emergency treatmentand these call for increased staff and facilities - they are part of the price of improving treatment and saving more desperate cases. Like the fast-moving traffic on the highway, the fast-moving illness of trauma can be handled most safely in a separate lane which is unobstructed by slower-moving problems.

Without doubt, the commonest mistake in the management of severe burns at present is the failure to make an adequate diagnosis. In no other branch of medicine do we so easily neglect physical signs or fail to look for them. Perhaps this is partly because so much of the skin surface is burned, and therefore untouchable by bare hands for fear of implanting infection. Again, if extensive burns are dressed, little of the patient can be examined except when they are re-dressed. Neither of these difficulties is insuperable, and I think that the chief reason for our failure to assess burned patients adequately is a genuine lack of appreciation of the importance of diagnosis. For this reason it is perhaps worth while to consider the management of severe burns in a way which will answer the question, "What should we look for, and how should we look for it?"

\section{Diagnosis of a "Shock Case"}

Most of the severely burned patients in this country reach hospital an hour or two after injury, and so the emphasis in treatment is on the prevention rather than the correction of shock. For prevention one must be able to diagnose a shock case before the complication occurs. Fortunately the plasma loss which is responsible for shock is roughly proportional to the extent of the body surface involved, and it is now established that if a burn extends over more than about $10 \%$ of the body surface in a child, or more than $15 \%$ in an adult, the patient will generally become clinically shocked after a few hours if the plasma loss is not replaced by colloid infusion. To diagnose the shock case one therefore needs to bear in mind these critical figures and some method of assessing body surface such

* Based on a communication read to the Section of Accident Surgery at the Annual Meeting of the British Medical Association, Birmingham, 1958. as the "rule of nines" (see Diagram). Although the rule of nines is only a rough guide, it is adequate for this assessment provided one bears in mind that a correction is needed for children, in whom the head forms a larger proportion of the body surface and the legs correspondingly less. To assess the body surface of a child's head and neck one should take the age of the child from the number 12 , and add the result to the figure for the adult head and neck $(9 \%)$; the result of the subtraction should be taken away from the combined surface-area figure for both legs (adult $38 \%$ ). Another measure is the patient's outstretched palm and fingers, which comprise $1 \%$ of the body surface.

Blood pressure is a late and very unreliable diagnostic guide to burn shock. A patient can lose $25 \%$ of his blood volume, an amount sufficient to cause serious renal damage, without his blood pressure falling. This does not mean, of course, that the blood pressure should

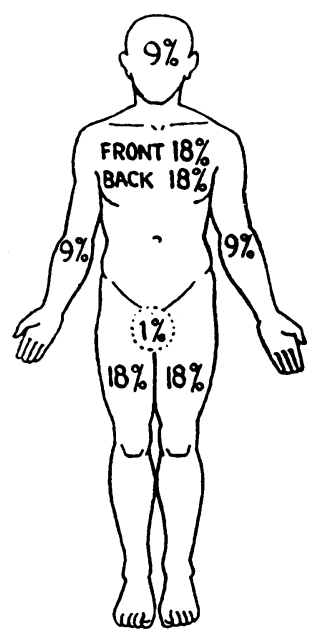

"Rule of nines" diagram for estimating area of burn. not be taken. It should always be taken at intervals when possible, but by the time it begins to fall the patient is well advanced in the stage of latent oligaemia, and may even be suffering from obvious clinical shock.

\section{Diagnosis of the Colloid Requirement}

The second question which must be answered is: "How much colloid should be given, and how fast?" It is a tragic admission, but unfortunately true, that rule-of-thumb methods are much too widely used in the resuscitation of burned patients. This is no criticism of the methods themselves. They have a valuable place in the treatment of mass casualties and similar emergencies, but to give individual patients in peacetime average requirements is inexcusable, even if it means that the doctor can leave the treatment of the patient to a nurse while he gets on with other work or goes to bed.

There are at least five good guides to the colloid requirement of the burned patient, and we cannot afford to be without any of them.

1. Physical Signs.-If the patient is pale, cold, restless, and with collapsed peripheral veins, these signs indicate oligaemia, and further colloid is required.

2. The Haematocrit.-The haematocrit level of the patient before his accident is of course unknown, but there are tables of average values according to age and sex, and initially the patient can be assumed to have an average value unless his first reading on admission is lower than this (see Table). Naturally, one must bear in mind that the patient may have been anaemic before his accident, but in this country one can rule out in this connexion the possibility of polycythaemia. From the initial haematocrit estimation, taken when the infusion is set up a few minutes after admission, the doctor can work out how much plasma has been lost if he assumes that the patient has a normal red-cell volume. This amount of plasma, together with the amount that is going to be lost in the next hour, should then be given in the first hour of infusion, so that by the end of this time the patient's blood volume is returned to normal. If the patient was anaemic before his accident 
Table Showing Average Normal Values. The Venous Haematocrit Values have been Corrected for Trapped Plasma by Subtracting $2 \%$ if the Centrifuge Haematocrit is $20-29 \%, 3 \%$ if Between 30 and $49 \%$, and $4 \%$ if Between 50 and $69 \%$. The Body Haematocrit Level is the Proportion of the Body Redcell Volume to the Total Blood Volume: In Practice it may be Calculated by Multiplying the Corrected Venous Haematobe Calculated by
crit bal by 0.9

HEIGHT WEIGHT AGE HAEMATOCRIT R.B.C.V. P.V. TOTAL B.V.

(cms) (kams) (rrs) venOUs Bopr

* venous haematocrit corrected for trapped plasma (55minutes $3000 \mathrm{r} \mathrm{P.} \mathrm{m.)}$

an insufficient amount of colloid will have been given, and perhaps he will still show signs of shock at the end of an hour's resuscitation; in this case the haematocrit level should be brought 5-10 points below normal in the next hour. In practice, too much colloid will never be given in the first hour if the haematocrit is simply brought back to the average normal for age and sex.

3. Rate of Urine Flow.-If the burn covers more than $20 \%$ of the body surface a urethral catheter is advisable to obtain an hourly figure for urine output. Usually it is not put in place until after the infusion has been set up, so that the bladder is not emptied for perhaps half an hour after admission and the hourly urine output is not known for a further hour after this. This guide is therefore of little value until the second hour. A urine output of 35 to $60 \mathrm{ml}$. an hour for an adult may be regarded as satisfactory ; this is $60-100 \%$ of the normal output. Corresponding amounts for children of 3-5 years, who are so commonly burned, are 15 to $25 \mathrm{ml}$. As mentioned above in connexion with the haematocrit, comparison with average figures can be misleading: the patient may develop some degree of renal insufficiency as a result of prolonged oligaemia, and this may be either oliguric or non-oliguric in type ; nevertheless, for most cases the hourly urine flow is most valuable.

4. A Formula.-There are many formulae giving the average colloid requirement for patients of known body weight and size of burn. Some are more complicated than others, but all of them are a rough measurement only. A patient's colloid requirement judged by using all the guides together may vary from the formula assessment by as much as $100 \%$ or more. The formula I prefer, which has the advantage of being simple, is that a patient will require an amount of colloid equal to his plasma volume for every $15 \%$ of the body surface burnad, excluding erythema. To use this formula one needs to know the average plasma volume for the patient's height, or height and weight (see Table). This formula gives the colloid requirement of the patient for the first 24 to 36 hours. About half this amount should be given in the first eight hours and the rest during the subsequent 16 to 28 hours of the shock period. This is a formula for colloid replacement only; further fluid must be given to meet the water loss from the kidneys, skin, and lungs, either by mouth or intravenously, if the patient is vomiting.

5. Estimations of Blood Volume.-The four guides given above do not always point in the same direction, and when doubt remains about the rate at which colloid should be given, a fifth guide-estimation of patient's blood volumebecomes the decisive factor. There are several ways in which the blood volume can be estimated, but the best at present is probably by a direct estimation of the red-cell volume using radioactive phosphorus or chromium. In a burns unit, facilities to get this estimation done rapidly and to have the answer in one to two hours should always be available.

No one of the guides mentioned is good enough to use alone, but they should all be considered together and the trend of them followed.

\section{Diagnosis of Destruction of Red Cells}

Red cells are destroyed in severe burns by loss from the body as haemorrhage, by destruction by heat in the burn, and by subsequent lysis in the circulation by a process which is still ill-understood.

As in the case of plasma loss, there are five useful guides to the amount of red-cell destruction.

1. Visible Evidence.-Red cells may be lost from the body in the urine as haematuria or haemoglobinuria. The patient may have a haematemesis, coffee-grounds vomit often being produced even where there is no definite ulcer in the stomach or duodenum. Sometimes there may be a melaena, and, rarely, haemolytic jaundice.

2. A Count of Fragmented Red Cells.-In addition to these obvious signs of blood loss there are accessory investigations which are valuable. Fragmented red cells may be seen and counted in a blood film taken on admission to hospital (Topley and Jackson, 1957). About half the cases which have more than $2 \%$ of heat-fragmented red cells in the head of the film have moderate red-cell destruction-that is, more than $25 \%$ of the red-cell volume destroyed in the first $\mathbf{4 8}$ hours.

3. Signs of Shock with a Normal Haematocrit Level.-A third sign of red-cell destruction is the persistence of physical signs of shock, such as pallor, restlessness, and a cold nose, forehead, and extremities, after the haematocrit level has been brought back to the average normal figure by colloid infusion. If the patient is still shocked at this stage, either he was anaemic before the accident or red cells were destroyed after the burn.

4. Falling Haematocrit Level in Absence of Over-infusion with Colloid.-A fourth guide is a low and steadily falling haematocrit level which is not due to over-infusion with colloid; this may be seen the same hour as the onset of haemoglobinuria.

5. Estimation of Red-cell Volume.-This is the same as the fifth guide to the correct colloid requirement-an estimation of the patient's individual red-cell volume.

Because all these guides are rough ones, none should be taken separately, but in conjunction with the others. Even the estimation of the red-cell volume can be deceptive. It is not only subject to the errors of the method, but there is evidence also that stagnation of red cells occurs somewhere in the body during the shock stage in $20-40 \%$ burns, and there may be subsequent remobilization of almost $20 \%$ of the red-cell volume at the end of this period. Owing to this factor the estimation of the blood volume may have an error of $\pm 20 \%$. 
The size of the burn has been purposely left till last. It may be asked whether there is not a simple and useful correlation between the size of deep burning and the degree of red-cell destruction. Unfortunately there is only a rough relationship, and the red-cell destruction in extensive burns is characterized more by variability in the amount and time of red-cell destruction than by correlation with size. Replacement of red cells by a formula may therefore be too little or too much.

In the absence of facilities for measuring the redcell volume, however, the rough correlation is an available second-best. If a burn is less than $20 \%$ of the body surface the red-cell destruction will probably be less than $10 \%$ of the red-cell volume. If the burn is 20 to $40 \%$ of the body surface, with an average amount of deep burning in it, the red-cell loss is likely to amount to about $20 \%$ of the red-cell volume. If the burn is more than $40 \%$ of the body surface, the redcell destruction may vary widely between 20 and $70 \%$ of the red-cell volume, sometimes occurring mostly in the first day and sometimes in the second.

It should be emphasized, of course, that keeping the red-cell volume normal except in cases of massive redcell destruction is of little importance compared with maintaining the blood volume at near normal levels. Knowledge of the red-cell volume and haematocrit level provides a blood-volume figure which is of great value when other guides are conflicting. It is particularly valuable, too, in oliguric renal failure when one wishes to give as little fluid as possible while being sure that enough colloid has been infused to prevent further ischaemic renal damage.

\section{Diagnosis of Renal Insufficiency}

Oliguria in burns shock may be physiological or pathological: it may be the result of oligaemia or of renal damage. In some conditions the specific gravity of the urine might be regarded as an index of renal function, even a small amount of concentrated urine suggesting no serious renal damage. In severe burns, however, glycosuria is often present in the first one or two days, and if dextran has been used for colloid replacement this may also be excreted in the urine in sufficient amounts to give a high specific gravity. For these reasons the concentration is more usefully determined by the freezing-point.

A better early test of renal function is the water tolerance test (Cope and Moore, 1947). In the presence of oliguria due to oligaemia a rapid intravenous infusion of a half to one litre of $5 \%$ dextrose in water will produce a brisk response in renal output in the next hour. If the renal output is not increased this is evidence of renal damage.

A large hourly urinary output is not a sure indication that all is well. It may be due to over-transfusion, or it may be due to the non-oliguric type of renal failure in which the kidney passes much water but with little in it. The guides of renal function which should be used routinely after the shock stage are estimations of blood urea, which should be carried out on the second, fourth, and sixth days after extensive burning, and daily checks on the urine volume, urea concentration, and osmolarity. With these it should be possible to diagnose renal insufficiency within 24 hours of its onset, and to alter treatment according to whether renal failure is oliguric or non-oliguric in type.
Oliguria is sometimes encountered after the shock stage, between the third and seventh days; although this may be due to renal failure it may also be due to dehydration. It is perhaps not sufficiently appreciated that as much as two litres a day of exudate may be lost from an adult's extensive burns, so that if the correct fluid intake is estimated without this factor the patient may be several litres of water short by the end of the first week. Loss of elasticity of the skin is a good sign of dehydration, and the diagnosis must not be excluded because the veins are full or because there is some residual oedema.

\section{Diagnosis of Electrolyte Imbalance}

When oral feeding is started on the second day and renal function is normal, there need be little concern about electrolytes. But if oral feeding has been impossible owing to vomiting, or if there is evidence of inadequate renal function, daily blood chemistry examinations are necessary.

Sodium Deficit.-A low serum sodium level is common between the third and seventh days in burned patients who are progressing favourably. It is not a sign of sodium deficit ; in fact, there is a positive sodium balance in the body as a whole due to the colloid (and saline) infusion which has formed the oedema. If normal feeding is not started at the end of the shock stage the serum level may fall to $115-130 \mathrm{mEq} / 1$. In this condition any augmenting factor, such as even slight overhydration with intravenous $5 \%$ glucose, may precipitate convulsions or coma.

Sodium Excess.-This is rarely seen with the treatment advised above when dried plasma is reconstituted with $400 \mathrm{ml}$. of water (sodium concentration 165 $\mathrm{mEq} / 1$.). However, hypernatraemia (serum sodium $>160 \mathrm{mEq} / \mathrm{l}$.) has been seen in large burns during the stage of absorption of oedema when non-oliguric renal failure is present with water diuresis and sodium retention. Treatment must then steer between dehydration, which is likely to increase the serum sodium level further, and hydration, which may cause pulmonary oedema.

Potassium Deficit.-The burned patient usually maintains a normal daily potassium excretion, and, provided adequate feeding is started on the second or third day, he will not develop a serious deficit. If, however, feeding is impossible, sufficient potassium should be given intravenously to cover the loss in the urine. This may be about $50 \mathrm{mEq}$ a day for an adult and $30 \mathrm{mEq}$ for a child, but it should be measured in the 24-hour collections of urine. It is well known that serum potassium levels are valueless as an index of the potassium balance in the body, because it is held for the most part in the tissues. This tissue reservoir is usually heavily drawn upon before the serum level falls.

Potassium Excess.-This is found in burned patients only when the potassium output is blocked by oliguric renal failure. A rise in serum potassium follows, but again it is a poor index of the balance in the tissues. In this condition every means must be used to prevent potassium intoxication by restricting intake, giving adequate glucose with insulin, and employing at the right time the methods available for potassium withdrawalfor example, ion-exchange resins and the artificial kidney. Even where the serum potassium level is kept within normal limits death may still occur from renal failure. 


\section{Diagnosis of Bacterial Colonization and Infection}

Bacteriological diagnoses in burned patients are often urgent and vital. Survival may depend on knowing the variety of pathogens which colonize the burn surface, whether invasive infection is present, whether there is a positive blood culture, and if so whether it signifies a transient bacteriaemia or septicaemia. In pulmonary infection the identity of the predominant organism is required and its sensitivity. In addition a decision must be made on the choice of local treatment for each burn.

Bacterial Colonization.-Diagnosis of the flora colonizing the burn should be made frequently so that the patient can be isolated and appropriate treatment given if important pathogens are found. By "important pathogens" one means those harmful to the "take" of skin grafts or resistant to most available antibiotics. Group A $\beta$-haemolytic streptococci are the most damaging to graft-take, with Pseudomonas pyocyanea and coliform bacilli second; Staphylococcus aureus and Groups $C, D$, and $G \beta$-haemolytic streptococci are less important in this respect. Although in the past some surgeons have belittled the importance of bacterial colonization on the grounds that some degree of it is usually inevitable, this is a quite unrealistic attitude, as much can be done to limit the amount of bacterial growth. Moreover, there is no chance of invasive infection unless the burn is first colonized.

Invasive Infection.-Pain is the principal sign of invasive infection in burns. Redness of the wound edge, excessive discharge, and pyrexia are also significant, but less so. In the exposure treatment of burns the surgeon should not be lulled into a sense of false security by reports of sterile swabs from the dry surface of the eschar ; the under surface may at the same time be bathed in pus containing many organisms.

Positive Blood Cultures.-The frequency with which positive blood cultures are found in burns increases with the thoroughness of the search for them. What do they mean? If a bacteriaemia occurs after dental extraction, how much more frequent must it be when a patient is lying on an extensive granulating wound. A patient with a positive blood culture is not always clinically ill, and it is possible that the positive result in these cases signifies a bacteriaemia only. At present, however, a septicaemia should be diagnosed and treated with all seriousness when a burned patient has a positive blood culture with a pathogenic organism. A quick bacteriological investigation is called for to diagnose the organism and assess its sensitivity, as the patient's life is at stake.

Pulmonary Infections.-One of the chief difficulties in treating pulmonary infections in burned patients is to find the predominant organism in the lungs so that the condition may be treated with the appropriate antibiotic. This is often an organism which has first colonized the burn. A post-nasal swab usually gives the same predominant organism as that found in the lungs, and we have used this method of diagnosis for several years.

Local Treatment.-The choice between dressings and exposure for local treatment demands an independent assessment for every patient. If practised efficiently both methods are good, and both practised negligently or without experience can be very bad. The surgeon should assess for each burn site in each patient the method which will give the best result in this particular circumstance. Factors to be considered are the time since injury and the condition of the burn. A recent clean burn on a limb can often be better protected from infection by an efficient closed dressing. On the other hand, an infected burn with excessive discharge and contaminated with Ps. pyocyanea can often be greatly improved in a few days by exposure and drying. Some parts of the body, such as the limbs and trunk, can be efficiently covered with dressings; other sites, such as the face, buttocks, and genitalia, are very difficult to dress efficiently and are better left exposed. Further factors are the facilities for doing dressings, the temperature of the environment, and the privacy of the patient's accommodation. There is no reason why both methods should not be employed in the same patient at the same time ; for instance, the face may be treated exposed, and both hands and arms dressed and elevated with the fingers held in the position of optimum function in dressings.

\section{Diagnosis of the Depth of Burning}

This is one of the most important early diagnoses that should be made. If the general practitioner can diagnose that the burn is partial thickness skin loss only, he can dress the burn himself, confident that healing will be complete in three weeks without scarring. If the surgeon can diagnose full-thickness skin destruction he can excise and graft the burn on the day of injury. Although experience is valuable we are not without helpful criteria which can be passed on by word and picture.

Perhaps the first lesson the clinician must learn is the deceptiveness of the appearance of burns. A white or charred burn, especially on the face, may turn out to be partial-thickness skin loss and be healed in a fortnight without scarring. On the other hand, a burn may be red, appearing little different from the erythema of a superficial burn, and yet turn out to be fullthickness skin loss. Although the appearance of a burn changes during the next few days and weeks, no new appearance or physical sign appears during the first week which makes diagnosis easier than it was on the first day.

There are usually three zones present in a typical fresh burn, and these zones indicate three intensities of burning. The outer one is the zone of hyperaemia. which is red and blanches on pressure, the colour returning with release. This zone remains red, with normal circulation, and by the end of a week is dry and re-epithelized. The middle zone, or zone of stasis, has a circulation for a period after injury, but within a matter of minutes or hours the circulation ceases and the capillaries are left stuffed with red cells. At first this zone is bright red and blanches on pressure, but when the circulation ceases it becomes mottled red and white and no longer blanches. The central zone is white, or even charred, and is the zone of coagulation. One should think of these three zones in depth as well as on the skin surface. According to the duration and intensity of burning, they may be shallow or deep although presenting the same surface appearance. The appearance of the burn informs about the upper surface of the skin; the only part of it we are anxious to know about is its deep surface.

Fortunately the sensitivity of the skin to pin-prick is helpful in determining the viability of the deeper layers of the skin. If, in this test, the patient can feel repeated firm pin-pricks as pressure only, analgesia is present. The interpretation of the test is this: if the 
patient can appreciate pin-prick as sharp, the burn is partial-thickness skin loss only, and it will heal without grafting in less than three weeks and leave no scar. The converse is not always true: if the burn is analgesic to pin-prick the burn is not necessarily full-thickness skin loss. It is either deep partial- or full-thickness skin loss. Even this knowledge is valuable to the surgeon. The skin in some regions, such as the dorsum of the hand or foot, heals badly after deep partial burning. In such cases excision and replacement with a thick split skin graft may be better.

There are exceptions to the sensitivity test. The face and scalp, and the palms and soles, have epithelial elements penetrating more deeply into the subcutaneous tissue than other parts of the body, and the pin-prick test should never be used in these areas. Similarly, it should not be used where the burn has been produced by chemicals, such as phenol, which have an analgesic action. It goes without saying that this test, like all the others mentioned earlier, should never be used in isolation, but in conjunction with knowledge of the anatomy of the skin and the history of the burn.

Yet another valuable sign in diagnosing the depth of necrosis when excising a deep burn is the red fat in the deeper part of the zone of stasis. This fat is dead. For three or four days after burning the zone of stasis in the skin and fat remains red, but after this time the red cells packing the capillaries lyse, and the avascular fat once more becomes white and difficult to distinguish from the normal fat beneath it.

\section{Summary}

The diagnostic problems of burns are discussed in this paper in the order in which they are usually encountered clinically.

The patient requiring resuscitation with colloid infusion may be diagnosed by the proportion of skin surface burned.

The amount and rate of colloid infusion may be assessed from hour to hour by following the changing trend of five clinical guides taken together-the general condition of the patient, the haematocrit level, the urine output, a formula, and measurement of the blood volume.

Whether blood transfusion is required depends on the degree of red-cell destruction. Although this varies greatly from case to case, both in amount and in time, it may also be assessed by five guiding factors-visible blood loss, evidence in a blood film, signs of shock with a normal haematocrit level, a falling haematocrit level without over-infusion, and direct measurement of the red-cell volume.

A routine is suggested for the early diagnosis of renal insufficiency; this needs to be differentiated from the physiological oliguria which accompanies oligaemia in the shock stage, or dehydration in the subsequent week. Oliguric and non-oliguric types of renal failure must also be distinguished if appropriate treatment is to be given.

The two criteria for suspecting electrolyte imbalance and for making daily blood chemistry examinations are renal insufficiency and failure to start oral feeding on the second day. Some suggestions are made for avoiding serious deficit or excess.

The rationale behind frequent bacteriological examination of the burn flora is given, and the early diagnosis of invasive infection and the choice of local treatment for the burn are discussed.

Finally, in connexion with the diagnosis of the depth of burning, the deceptiveness of appearance and the value of sensitivity to pin-prick as a sign of partialthickness skin loss are emphasized.

Constant reassessment of the changing condition of the patient is an essential feature of successful burns treatment. Sometimes it can be measured, sometimes it must be an informed guess; but always the surgeon must be seeking to forestall the likely complications and to recognize in time their small beginnings.

\section{REFERENCES}

Cope, O., and Moore, F. D. (1947). Ann. Surg., 126, 1010. Topley, E., and Jackson, D. MacG. (1957). J. clin. Path., 10, 1.

\section{PREDNISOLONE TRIMETHYLACETATE IN INTRA-ARTICULAR THERAPY}

$$
\text { BY }
$$

W. R. MURDOCH, M.B., F.R.F.P.S., M.R.C.P.Ed. Medical Registrar, Glasgow Royal Infirmary

AND

\author{
G. WILL, M.B., B.Sc., F.R.F.P.S. \\ Senior Medical Registrar, Glasgow Royal Infirmary
}

Since the introduction of the steroids there has been a renewed interest in intra-articular therapy. The compound most often used has been hydrocortisone, and there is a voluminous literature on its effects. Those authors who have carried out controlled trials (Dixon and Bywaters, 1953 ; Hollander, 1953 ; Duff et al., 1955 ; Duff, 1956 ; Fearnley et al., 1956) are in agreement that hydrocortisone produces symptomatic benefit in many cases, but several authors have found this to be variable. Thus Robinson et al. (1955) obtained worth-while benefit in only half of the joints, although treatment was combined with special orthopaedic and physical therapy measures. Duff (1956) gained worth-while benefit of 7 to 10 days' duration on the average in $50 \%$ of cases, while in $31 \%$ response was poor, and there was no significant response in $19 \%$. Sairanen (1956) obtained the best results in finger-joints. In the knee-joints the results were poor in $44 \%$. Increased benefit was not obtained by large doses of up to $100 \mathrm{mg}$. or from repeated injections at short intervals. He concluded that new products with a prolonged effect would doubtless be the solution.

The desirable compound for intra-articular therapy is one with a marked anti-inflammatory action but low solubility. In this way an effective local concentration will be maintained for a prolonged period with little systemic absorption. A number of compounds have been derived from prednisone and prednisolone, the delta-1 derivatives of cortisone and hydrocortisone respectively, which have on systemic administration a greater anti-inflammatory activity than that of the parent substances. As prednisolone is the corresponding compound to hydrocortisone it would appear to be the more suitable for intra-articular therapy. The acetate esters of these compounds are of low solubility. The use of suspensions of crystals of fixed dimensions further delays solution. From a very large number of esters of prednisone and prednisolone investigated, 